\title{
Possibility Semantics for Intuitionistic Logic
}

\author{
M. J. Cresswell \\ Department of Philosophy \\ University of Auckland \\ Deparment of Philosophy \\ Texas A\&M University \\ m-cresswell@philosophy . tamu .edu \\ Received by Greg Restall \\ Published April 30, 2004 \\ http://www.philosophy.unimelb.edu.au/ajl/2004 \\ (C) 2004 M. J. Cresswell
}

\begin{abstract}
The paper investigates interpretations of propositional and firstorder logic in which validity is defined in terms of partial indices; sometimes called possibilities but here understood as non-empty subsets of a set $W$ of possible worlds. Truth at a set of worlds is understood to be truth at every world in the set. If all subsets of $W$ are permitted the logic so determined is classical first-order predicate logic. Restricting allowable subsets and then imposing certain closure conditions provides a modelling for intuitionistic predicate logic. The same semantic interpretation rules are used in both logics for all the operators.
\end{abstract}

In standard modellings of intuitionistic logic, disjunction $(V)$ is usually understood as classical, while negation $(\sim$ or $\neg$ ) is understood non-classically. In this paper I present a semantics for intuitionistic logic according to which it is disjunction, rather than negation, which is not classical. I call it 'possibility semantics', following Humberstone I98I and Chapter 8 of Cresswell I990, because it treats the indices at which formulae are evaluated for truth and falsity as 'partial' or 'incomplete' indices. One way of understanding an incomplete index is in the sense of a proposition, where a proposition is thought of as a class of possible worlds. In looking at intuitionistic logic from a classical perspective it is natural to follow the Kripke modelling and interpret intuitionistic negation $\neg$ as something like $\square \sim$, where $\square$ is the necessity operator and is classical negation, together with a restriction on the value assignments to the propositional variables. I shew how to give a unified semantics for both $\sim$ and $\checkmark$ which gives classical or intuitionistic logic according to different restrictions placed on the 'allowable' indices. The philosophical importance of this is that you can then exhibit the difference between the two logics not as a matter of 
what you choose to mean by 'not' or any other operator, but as a matter of how the world presents itself for evaluation in terms of truth. T]

So assume a set $\mathrm{W}$ of possible worlds and assume a value assignment $\mathrm{V}$ which assigns to every variable in a standard propositional language a set of possible worlds. Assume that the language contains $\sim, \wedge, \supset$ and $\vee$. Where $w$ is a possible world we may define truth at $w$ in the obvious way, where $\vDash$ is the 'truth predicate' which depends on $\mathrm{V}$ :

$$
\begin{aligned}
& \vDash_{w} p \text { iff } w \in V(p) . \\
& \vDash_{w} \sim \alpha \text { iff } \exists_{w} \alpha\left(\text { where ' }=\text { ' means 'not } \vDash^{\prime}\right) . \\
& \vDash_{w} \alpha \wedge \beta \text { iff both } \vDash_{w} \alpha \text { and } \vDash_{w} \beta . \\
& \vDash_{w} \alpha \supset \beta \text { iff either } \exists_{w} \alpha \text { or } \vDash_{w} \beta . \\
& \vDash_{w} \alpha \vee \beta \text { iff either } \vDash_{w} \alpha \text { or } \vDash_{w} \beta .
\end{aligned}
$$

Call a wff valid iff it is true at every index in every assignment. The class of valid wff is of course just the classical propositional calculus. What then about truth at a proposition? Assume the (classical) 'adequacy requirement' that a wff is true at a (non-empty) set $a$ of worlds iff it is true at every $w \in \mathrm{a}$. ' $\alpha$ is true at a' can be written as $\vDash_{a} \alpha$ and the requirement can be stated as:

$$
\text { [A] } \vDash_{\mathrm{a}} \alpha \text { iff } \vDash_{w} \alpha \text { for all } w \in \text { a. }
$$

For propositions of the form $\{w\}$, i. e propositions consisting of a single world, the rules given above can be stated with $\vDash_{\{w\}}$ in place of $\vDash_{w}$. It is a consequence of requirement $[\mathrm{A}]$ that negation at a set of worlds does not satisfy the standard truth table. For suppose $a=\left\{w_{1}, w_{2}\right\}$, and $V(p)=\left\{w_{1}\right\}$. Then, by $[\mathrm{A}], \exists_{a} p$, since $w_{2} \in a$ and $\exists_{w_{2}} p$, and $\exists_{a} \sim p$ since $w_{1} \in a$ and $\exists_{w_{1}} \sim p$. But despite this $[\mathrm{A}]$ gives us a completely classical logic in the sense that all classically valid wff of this language will be true at every possible world and so true at every set of worlds..$^{2}$

I shall now investigate what happens if in place of the truth rules given in terms of possible worlds and then applied to truth at propositions via requirement $[\mathrm{A}]$ we try to define directly truth at a proposition. Say that $\langle\mathrm{W}, \mathrm{V}\rangle$ is a tractarian $\sqrt{3}$ model iff $W$ is a set (of 'worlds') and for every propositional variable

${ }^{I}$ There are of course many discussions of the semantics of intuitionistic logic, and I am able to do no more than allude in passing to some of them. For instance, comments on an earlier version of this paper suggested that its results were well known as the 'Beth-Kripke-Joyal semantics', as reported in theorem 4.39 on p. 156 of Bell I988 or in Theorem 8.4 on p. 166 of Lambek and Scott 1986. It is true that the Beth-Kripke-Joyal semantics depends on a non-standard semantics for $\vee$ which gives classical logic in standard set theory, and gives intuitionistic logic under other conditions. However, I shew below that while there may be some connection between the Beth-Kripke-Joyal semantics and the results obtained here it does not seem to be a direct connection. (I am grateful to Lloyd Humberstone for encouraging me not to lie down and play dead when I was accused of re-inventing the wheel!)

${ }^{2} \mathrm{~A}$ principle like $[\mathrm{A}]$ in the context of tense logic with indices as intervals is mentioned on p. 42 of van Benthem 1985 .

3I use the word 'tractarian' to acknowledge the influence of Wittgenstein I92I on at least one way of understanding incomplete indices. I discuss this at the end of the paper. 
$p, V(p) \subseteq W \llbracket(V(p)$ may be empty.) Where $\emptyset \neq a \subseteq W$ we define the truth of a wff $\alpha$ at $a$, written $\vDash_{a} \alpha$, as follows. For atomic wff we have

$[p] \vDash_{a} p$ iff $a \subseteq V(p)$.

(In strictness I should write $V \vDash_{a} \alpha$ to indicate the dependence of $\vDash$ on $V$, but throughout the paper I shall suppress this where it is obvious which model is involved.) The rule for conjunction poses no problems:

$$
[\wedge] \vDash_{a} \alpha \wedge \beta \text { iff } \vDash_{a} \alpha \text { and } \vDash_{a} \beta .
$$

For $\sim$, requirement $[\mathrm{A}]$ would make $\sim p$ true at a iff $p$ is false at every $w \in$ a. In terms of propositions this can be stated as

$$
[\sim] \vDash_{\mathrm{a}} \sim \alpha \text { iff } \vDash_{\mathrm{b}} \alpha \text { for every } \emptyset \neq \mathrm{b} \subseteq \mathrm{a} .
$$

Say that an operator $O$ is (classically) respectable iff, provided $\alpha_{1}, \ldots, \alpha_{n}$ satisfy $[\mathrm{A}]$ then $\mathrm{O} \alpha_{1} \ldots \alpha_{n}$ satisfies [A]. It is an obvious consequence of this definition that if all atomic wff satisfy $[p]$ and all the operators are respectable then all wff satisfy $[\mathrm{A}]$. Further, if all operators are truth-functional at worlds, then the class of wff true at every index in every interpretation is just the classical propositional calculus. Validity of course now means truth at every non-empty set of worlds in every tractarian frame. If $[\mathrm{A}]$ is in force then [ ] emerges as a completely classical account of negation for the following reason: Suppose $\vDash_{a} \sim \alpha$. Then $\exists_{b} \alpha$ for all $b \subseteq a$. In particular, if $w \in a$ then $\{w\} \subseteq a$ and so $\exists_{w} \alpha$ and so $\vDash_{w} \sim \alpha$ for all $w \in a$. Suppose $\exists_{a} \sim \alpha$. Then for some $b \subseteq a$, $\vDash_{\mathrm{b}} \alpha$. But if $\alpha$ satisfies [A] then for any $w \in \mathrm{b}, \vDash_{w} \alpha$ and so $\exists_{w} \sim \alpha$. But $w \in \mathrm{b}$ and $\mathrm{b} \subseteq \mathrm{a}$, and so for some $w \in \mathrm{a}, \exists_{w} \sim \alpha$.

The rule for implication is

$$
[\supset] \vDash_{a} \alpha \supset \beta \text { iff for every } b \subseteq \text { a either } f_{b} \alpha \text { or } \vDash_{b} \beta .
$$

It is a consequence of [つ] that $\supset$ is respectable. If $\vDash_{a} \alpha \supset \beta$ and $w \in a$ then, by $[\supset], \exists_{w} \alpha$ or $\vDash_{w} \beta$ and so $\vDash_{w} \alpha \supset \beta$. Conversely, suppose $\exists_{a} \alpha \supset \beta$. Then, by $[\supset]$, for some $\mathrm{b} \subseteq \mathrm{a}, \vDash_{\mathrm{b}} \alpha$ and $\exists_{\mathrm{b}} \beta$. So there is some $w \in \mathrm{b}$ such that $\exists_{w} \beta$.

\footnotetext{
${ }^{4}$ I have stated things on the assumption that a 'propositional' index $\mathrm{a}$ is a set of worlds. That is why I have chosen to use the word 'proposition' rather than Humberstone's word 'possibility'. While my formulation of $[\mathrm{A}]$ requires the assumption of sets of worlds the case can be described without that assumption. It is important to do so since those who want to use 'incomplete' indices in this way often object to construing them as sets of complete indices. Following Humberstone $198 \mathrm{I}$ call $\mathrm{a}^{\prime}$ a refinement of $\mathrm{a}$, if $\mathrm{a}^{\prime}$ gives all the information that a gives, and perhaps more besides. If $a$ is a set of worlds, as I have been assuming in the text, then refinement is simply class inclusion. Humberstone I98I, p. 3I8, has a condition he calls refinability, that if neither $\alpha$ nor $\sim \alpha$ is true at an index then there are two refinements of that index with $\alpha$ true at one refinement and $\sim \alpha$ true at the other. Humberstone is concerned to obtain classical logic as the logic of possibilities, though Humberstone takes possibilities as primitive. I am particularly grateful to Lloyd Humberstone for extremely detailed and helpful comments on an earlier draft of this paper.
} 
But $w \in \mathrm{b}$ and $\vDash_{\mathrm{b}} \alpha$, so $\vDash_{w} \alpha$. So, for some $w \in \mathrm{b}, \exists_{w} \alpha \supset \beta$. But $\mathrm{b} \subseteq \mathrm{a}$ and so for some $w \in \mathrm{a}, \exists_{w} \alpha \supset \beta$. [D] in conjunction with a 'falsum' constant, $\perp$, true at no world, will ensure that $\alpha \supset \perp$ is equivalent to $\sim \alpha$ according to $[\sim]$.

Despite the fact that these operators have been described as leading to classical logic the semantics just presented has a close connection with intuitionistic logic.5 The standard Kripke semantics for intuitionistic logic is based on frames of the form $\langle I, R\rangle$ where $I$ is a set of indices and $R$ a reflexive and transitive relation on the indices. A model $\langle I, R, V\rangle$ places on $V$ the condition that if a propositional variable $p$ is true at an index $i$ and $i R j$ then $p$ is true at $j$. I have referred to an index $i$ rather than to a world, since 'index' is a neutral term. In a tractarian frame the indices are non-empty sets of worlds, but in the Kripke semantics nothing is said about what they might be. If you treat an index as a set of worlds - as what I have called a proposition - then you can say that $\mathrm{aRb}$ iff $\mathrm{b} \subseteq \mathrm{a}$. So any tractarian rule in which the conditions are stated using only $\subseteq$ has an analogue in a Kripke model for intuitionistic logic. In particular, the Kripke rules for $\Lambda, \sim$ and $\supset$ are the same as those given for tractarian models except that I write $V(\alpha, i)=1$ instead of $\vDash_{a} \alpha$ and $V(\alpha, i)=0$ instead of $f_{a} \alpha$. Specifically they are these:

$V(\alpha \wedge \beta, i)=1$ if $V(\alpha, i)=1$ and $V(\beta, i)=1$, and 0 otherwise.

$V(\sim \alpha, i)=1$ if $V(\alpha, j)=0$ for every $j$ where $i R j$ and 0 otherwise.

$V(\alpha \supset \beta, i)=1$ if, for every $j$ where $i R j$, either $V(\alpha, j)=0$

$$
\text { or } V(\beta, j)=1 \text {, and } 0 \text { otherwise. }
$$

In such a semantics one might regard the frame as setting the structure of logical space, while the valuation rules determine the meaning of the operators within that space. There is a trade-off here. A paper such as Došen I991 shews that the reflexive and transitive properties of $R$ can be omitted if we impose restrictions on the truth sets of wff (i. e. the sets of indices at which a given wff is true.) By contrast, the aim of the present paper is to shew that by taking the indices as sets of worlds and by adopting a non-standard semantics for $V$, you can get either classical or intuitionistic logic according to which sets you admit as indices. Notice though - and this will be important enough for me to repeat from time to time - that restricting which sets of worlds count as allowable indices is quite different from specifying which sets of indices count as allowable truth sets. A procedure closer in spirit to the present paper, but using Kripke frames, would be one which simply restricts Kripke frames to oneworld reflexive frames in order to get classical logic. Such a restriction is stated purely at the frame level. There is even a philosophical motivation for it in that a classical logician might be one who assumes that truth and falsity is a onceand-for-all matter, while an intuitionist sees it as a developing matter. However,

\footnotetext{
${ }^{5}$ In my discussion of intuitionistic logic I am relying primarily on van Dalen I986, pp. 246252. The paper is of course in the tradition of giving a 'classical' account of intuitionistic logic, but, unlike many other attempts to do this, it does not interpret intuitionistic logic by giving a meaning for $\neg$ and $\rightarrow$ different from the 'classical' meaning given to $\sim$ and $\supset$.
} 
one-world frames do not admit an explanation of the necessity of classical logic, and some classical logicians may therefore want more.

From the usual point of view intuitionistic negation and implication, frequently written $\neg$ and $\rightarrow$, are considered non-classical. By contrast disjunction in the Kripke semantics for intuitionistic logic is considered classical, since $\vee$ has the rule that $V(\alpha \vee \beta, i)=1$ iff $V(\alpha, i)=1$ or $V(\beta, i)=1$. The corresponding tractarian rule would be:

$$
[V] \vDash_{a} \alpha \vee \beta \text { iff either } \vDash_{a} \alpha \text { or } \vDash_{a} \beta
$$

But it is easy to see that when the indices are non-empty sets of worlds and $[\mathrm{A}]$ is in force then it is $[\mathrm{V}]$ rather than $[\sim]$ or $[\supset]$ which is not classically respectable. For let $\mathrm{a}=\left\{w_{1}, w_{2}\right\}$ and let $\mathrm{V}(\mathrm{p})=\left\{w_{1}\right\}$ and $\mathrm{V}(\mathrm{q})=\left\{w_{2}\right\}$. Then $\vDash_{\left\{w_{1}\right\}} p$ and so $\vDash_{\left\{w_{1}\right\}} p \vee q$ and $\vDash_{\left\{w_{2}\right\}} q$ and so $\vDash_{\left\{w_{2}\right\}} p \vee q$. So by $[A], \vDash_{a} p \vee q$. But $\exists_{\left\{w_{2}\right\}} p$ and $\left\{w_{2}\right\} \subseteq a$ and so $\exists_{a} p$ and $\exists_{\left\{w_{1}\right\}} q$ and $\left\{w_{1}\right\} \subseteq a$ and so $\exists_{a}$ q. So, by $[\vee]$ we have the contradictory result that $\exists_{a} p \vee q,{ }^{6}$

The following rule gives a semantics for $\vee$ which is classically respectable:

$\left[\mathrm{V}^{\cup}\right] \vDash_{\mathrm{a}} \alpha \vee \beta$ iff there are some $\mathrm{b}$ and $\mathrm{c}$ such that $\mathrm{a} \subseteq \mathrm{b} \cup \mathrm{c}$ and $\vDash_{\mathrm{b}} \alpha$ or $\vDash_{\mathrm{b}} \beta$, and $\vDash_{\mathrm{c}} \alpha$ or $\vDash_{\mathrm{c}} \beta \cdot 7$

${ }^{6}$ Note that $[\mathrm{V}]$ gives the same result as the rule that $\vDash_{\mathrm{a}} \alpha \vee \beta$ iff either $\vDash_{\mathrm{b}} \alpha$ or $\vDash_{\mathrm{b}} \beta$ for all $\mathrm{b} \subseteq \mathrm{a}$. There is an alternative semantics for $\vee$ due to E. W. Beth. (See van Dalen I986, pp. 246-252.) Say that a path is a set $C$ of indices such that for any $a, b \in C$ either $a \subseteq b$ or $\mathrm{b} \subseteq \mathrm{a}$, and if $\mathrm{C} \subseteq \mathrm{C}^{\prime}$ and for any $\mathrm{a}, \mathrm{b} \in \mathrm{C}^{\prime}$ either $\mathrm{a} \subseteq \mathrm{b}$ or $\mathrm{b} \subseteq \mathrm{a}$, then $\mathrm{C}=\mathrm{C}^{\prime}$. Then say that $\mathrm{B}$ is a bar for $\mathrm{a}$ iff $\mathrm{B} \subseteq \wp \mathrm{W}$ and for any path $\mathrm{C}$ such that $\mathrm{a} \in \mathrm{C}$ there is some $\mathrm{b} \in \mathrm{C}$ such that $\mathrm{b} \in \mathrm{B}$. What this means is that every path through a goes through $\mathrm{B}$.

$\left[\vee^{\text {Beth }}\right] \vDash_{a} \alpha \vee \beta$ iff there is some $B$ which is a bar for $a$, and for all $b \in B$, either $\vDash_{b} \alpha$ or $\vDash_{b} \beta$.

From an intuitionistic point of view the idea is that however the future develops at least one of $\alpha$ or $\beta$ will eventually be true. In some futures it might be $\alpha$ and in others $\beta$, but in all of them one at least will be true. But the Beth semantics fares no better than [V] from our point of view, since it is not hard to see that $\left[\mathrm{V}^{\mathrm{Beth}}\right]$ is not respectable. Let the indices be sets of worlds where we may take the 'worlds' to be natural numbers. Where $W$ is the set of natural numbers let $a_{0}=W$ and for $n>0$ let $a_{n}=a_{n-1}-\{n\}$. Let $C=\left\{a_{n}: n \geqslant 0\right\}$. Obviously if $n \leqslant m$ then $a_{m} \subseteq a_{n}$. C is clearly a path, and further, it is a maximal path, for suppose there is some chain $C^{\prime}$ such that $C \subseteq C^{\prime}$ but $C^{\prime} \neq C$. Let $b \in C^{\prime}$ but $b \notin C$. Then either (i) for some $n \geqslant 0$, $a_{n} \subseteq b \subseteq a_{n-1}$ and $b \neq a_{n}$ and $b \neq a_{n-1}$ or (ii) $b \subseteq a_{n}$ for every $n \geqslant 0$. If (i) then there is some $k$ such that $k \notin a_{n}$ and $k \in b$, and some $j \notin b$ and $j \in a_{n-1}$. So $k \in a_{n}$ and $k \notin a_{n-1}$ and $j \in a_{n}$ and $j \notin a_{n-1}$. But in that case $k=n=j$, which contradicts the assumption that $k \in b$ and $j \notin b$. Suppose $b \subseteq a_{n}$ for every $n \geqslant 0$. Since $b \neq \emptyset$ suppose $h \in b$. But $h \notin a_{h}$. Now let $V(p)=\{n: n$ is even $\}$. Then $\exists_{a} p$ for all $a \in C$ and $\exists_{a} \sim p$ for all $a \in C$, and so there is no bar $B$ for $a_{0}$ such that $\vDash_{b} p$ or $\vDash_{b} \sim p$ for all $b \in B$. So, by $\left[\vee^{\text {Beth }}\right], \exists_{a_{0}} p \vee \sim p$; and therefore $\left[\vee^{\text {Beth }}\right]$ is not respectable.

${ }^{7}$ Another rule might seem to be:

$\left[\mathrm{V}^{\prime}\right] \vDash_{\mathrm{a}} \alpha \vee \beta$ iff for all $\mathrm{b} \subseteq \mathrm{a}$ there is some $\mathrm{c} \subseteq \mathrm{b}$ such that $\vDash_{\mathrm{c}} \alpha$ or $\vDash_{\mathrm{c}} \beta$.

See p. 322 of Humberstone $198 \mathrm{I}$ and p. 238 of van Benthem 1986 for an equivalent condition. However, $\left[\mathrm{V}^{\prime}\right]$ is simply what you get when you define $\alpha \vee \beta$ as $\sim(\alpha \wedge \sim \beta)$, and will not therefore provide a semantics for the full intuitionistic logic, since, as is well known, the set of intuitionistically valid wff in $\sim$ and $\wedge$ is the same as those valid in classical logic. (See Gödel, I933, p. 34.) 
Theorem I If $\vee$ satisfies $\left[V^{\cup}\right]$ then $\vee$ is classically respectable.

Proof Suppose $\vDash_{a} \alpha \vee \beta$. Then there are some $b$ and $c$ such that $a \subseteq b \cup c$ and $\vDash_{\mathrm{b}} \alpha$ or $\vDash_{\mathrm{b}} \beta$, and $\vDash_{\mathrm{c}} \alpha$ or $\vDash_{\mathrm{c}} \beta$. Suppose $w \in \mathrm{a}$. Then $w \in \mathrm{b} \cup \mathrm{c}$ and so $w \in \mathrm{b}$ or $w \in \mathrm{c}$. If $w \in \mathrm{b}$ then $\vDash_{w} \alpha$ or $\vDash_{w} \beta$ and so $\vDash_{w} \alpha \vee \beta$. Likewise if $w \in c$. Now suppose that, for all $w \in a, \vDash_{w} \alpha \vee \beta$. First if $\exists_{w} \alpha$ for all $w \in a$ then $\vDash_{w} \beta$ for all $w \in a$, and so $\vDash_{a} \beta$, and so $\vDash_{a} \alpha \vee \beta$. (And mutatis mutandis if $\exists_{w} \quad \beta$ for all $w \in$ a.) Otherwise let $\mathrm{b}=\left\{w \in W: \vDash_{w} \alpha\right\}$ and $\mathrm{c}=\left\{w \in W: \vDash_{w} \beta\right\}$. Then $\mathrm{a} \subseteq \mathrm{b} \cup \mathrm{c}$ and $\vDash_{\mathrm{b}} \alpha$ and $\vDash_{\mathrm{c}} \beta$, and so, by [V $\cup$, $\vDash_{\mathrm{a}} \propto \vee \beta$.

One way to distinguish between classical and intuitionistic logic might therefore be by choosing which of $[\mathrm{V}]$ or $\left[\mathrm{V}^{\cup}\right]$ to use. But using different interpretation rules seems an admission that $\vee$ has different meanings, and in fact would permit a logic with two symbols, one governed by $[\mathrm{V}]$ and one governed by $\left[\mathrm{V}^{\cup}\right]{ }^{8}$ It is an assumption of the present paper that the operators should obey the same rules in both logics, but that the differences should emerge by considering the nature of the indices. Since $[A]$ demands respectable operators I shall assume that the semantic rule for $V$ is $\left[\mathrm{V}^{\cup}\right]$ and shall examine what must be done to obtain intuitionistic logic using $\left[\mathrm{V}^{\cup}\right]$. But first we need an important theorem:

Theorem 2 If $\vDash_{\mathrm{a}} \alpha$ and $\mathrm{b} \subseteq \mathrm{a}$ then $\vDash_{\mathrm{b}} \alpha$.

Proof The proof is by induction on the construction of wff. The theorem is defined to hold for atomic wff, and the rules for $\sim, \wedge, \supset$ and $\vee$ (whether $[V]$ or $[\mathrm{V}]$ is used) ensure that it holds for all wff.

The analogue of Theorem 2 holds in all Kripke models for intuitionistic logic. As a consequence, if $i R j$ and $j R i$ then for all $\alpha, V(\alpha, i)=V(\alpha, j)$, and so every equivalence class of indices may be reduced to one with just a single member. Thus any intuitionistic frame is equivalent to one in which $R$ is antisymmetrical.

The proofs that the operators defined by these conditions are respectable of course depend on the fact that the system of indices is a complete Boolean algebra, and Kripke frames for intuitionistic logic merely require that the accessibility relation be reflexive and transitive. In particular I have made essential use of sets of the form $\{w\}$ which have the property that $\exists_{\{w\}} \sim \gamma$ iff $\vDash_{\{w\}} \gamma$ for any wff $\gamma$. Put in terms of indices in Kripke frames this requires that for any $i$ there be some $j$ such that $i R j$ and $V(\sim \gamma, j)=1$ iff $V(\gamma, j)=0$ for any wff $\gamma$. So, in the first place every index $i$ can see an endpoint $j$ where if $j R k$

\footnotetext{
${ }^{8}$ Schulz 1993 p. 176 , in the context of situation theory, has two disjunction operators, one, written as $\cup$, behaves like $[V]$, i. e. 'classically', while the other is equivalent to $\sim(\alpha \wedge \sim \beta)$. On p. I84 Schulz appears to reject a condition which would state that if $\alpha \vee \sim \alpha$ fails at an incomplete index $a$ (Schulz has failing at an infon $\sigma$ ) then there are indices $b$ and $c$ such that $b \subseteq a$ and $c \subseteq \mathrm{a}$ and $\alpha$ is true at $\mathrm{a}$ and $\sim \alpha$ is true at $\mathrm{c}$. He calls this condition 'complete splitting', and claims it would trivialize the theory. This would seem to be a rejection of some such rule as $\left[\mathrm{V}^{\cup}\right]$.
} 
then $j=k$. In the second place the tractarian semantics would require models which satisfy the following endpoint property:

[EP] If $V(\alpha, j)=1$ for every endpoint $j$ such that $i R j$ then $V(\alpha, i)=1.9$

If all propositional variables satisfy $[\mathrm{EP}]$ and all operators behave classically on worlds and are respectable, then the logic so determined is classical PC. Now consider the following (Kripke) frame, which I shall call $F_{1}$ :

$$
I=\{i, j\} \text { and } R=\{\langle i, i\rangle,\langle i, j\rangle,\langle j, j\rangle\} .
$$

In $F_{1}, R$ is a partial ordering, and so the frame is a (Kripke) frame for intuitionistic logic. This frame will falsify $\sim p \supset p$ by making $p$ true at $j$ but false at $i$. From the latter $V(p, i)=0$ and from the former $V(\sim p, i)=0$ and $V(\sim p, j)=0$, and so $V(\sim \sim p, i)=1$. However, although every world in this frame can see an endpoint this model does not satisfy $[\mathrm{EP}]{ }_{\mathrm{H}}^{\mathrm{O}}$ Actually $\mathrm{F}_{1}$ is not a possible tractarian frame, since in a tractarian frame an endpoint, here $j$, is $\{w\}$ for some world $w$. $i$ cannot be an endpoint since $i \neq j$. So there is some $w^{\prime} \neq w$ with $\left\{w, w^{\prime}\right\} \subseteq i$. But then $\left\{w^{\prime}\right\} \subseteq i$ and since $j \neq\left\{w^{\prime}\right\}$ there must be some endpoint $k$ such that $i R k$ and $k \neq j$. Thus we get the following frame $F_{2}$ :

$$
I=\{i, j, k\} \text { and } R=\{\langle i, i\rangle,\langle i, j\rangle,\langle i, k\rangle,\langle j, j\rangle,\langle k, k\rangle\}
$$

i. e $i$ can see itself and the two endpoints $j$ and $k$. In terms of a frame with tractarian indices the worlds would be $w_{1}$ and $w_{2}$, and the indices would be $\mathrm{a}=\left\{w_{1}, w_{2}\right\}, \mathrm{b}=\left\{w_{1}\right\}$ and $\mathrm{c}=\left\{w_{2}\right\}$. But suppose we disallow $\mathrm{c}$ as a possible index. The rationale might be this. In understanding the meaning of a wff $\alpha$ we need to know its truth conditions - we need to know whether it is true at this or that index. Suppose that $\mathrm{c}$ is an index to which we have no epistemic access. $F_{1}$ could be thought of as the 'conscious' part of reality. Although $c$ is not present to consciousness, and therefore is not an index in $F_{1}$, yet it covers a part of reality which has effects that are so present. Putting $\mathrm{V}(\mathrm{p})=$ $\left\{w_{1}\right\}$, and therefore making $p$ false at $w_{2}$, is enough to stop $p$ being true at a despite the fact that $c$ is not an allowable index, since the falsity of $p$ at $w_{2}$ is discerned at $a$, which is an allowable index. (This is because a $\nsubseteq \mathrm{V}(\mathrm{p})$.) Thus an intuitionistic frame can be embedded in a tractarian frame by allowing

${ }^{9}$ There is a related property which van Benthem 1986 p. 238 , calls stability:

[S] If for all $k$ such that $i R k$ there is some $j$ where $k R j$ and $V(\alpha, j)=1$ then $V(\alpha, i)=1$.

Where every index can see an endpoint $[\mathrm{EP}]$ and $[\mathrm{S}]$ are equivalent. For wff of the form $\sim \alpha[\mathrm{S}]$ always holds, for suppose $i R k$. Then if there is some $j$ such that $k R j$ and $V(\sim \alpha, j)=1$ then, for every $h$ such that $j R h, V(\alpha, h)=0$. But $k R h$, and so, by Theorem $2, V(\alpha, k)=0$. If this is so for every $k$ such that $i R k$ then $V(\sim \alpha, i)=1$, satisfying $[S]$.

${ }^{10}$ In tractarian models, and in all Kripke models which satisfy [EP], $\alpha \supset \beta$ may be defined as $\sim(\alpha \wedge \sim \beta)$. But without $[\mathrm{EP}]$ this will not work for Kripke models in general. Take the following model based on $F_{1}: V(p, i)=V(p, j)=V(q, j)=1, V(q, i)=0$. Since $i R i$ then $V(p \supset q, i)=0$. But $V(q, j)=1$, and so $V(p \wedge \sim q, j)=0$ and $V(p \wedge \sim q, i)=0$. So $V(\sim(p \wedge \sim q), i)=1$. 
only certain sets of worlds ${ }^{m}$ Using this technique we can give a precise sense in which intuitionistic logic is just what you get from the logic of tractarian indices if you place restrictions on which sets of worlds are indices.

Say that a general tractarian frame is a pair $\langle W, P\rangle$ in which $W$ is a set (of 'worlds') and $\mathrm{P}$ is any non-empty set of non-empty subsets of $W$; i. e. $\emptyset \neq \mathrm{P} \subseteq$ $\wp W-\emptyset$. $P$ is the set of 'allowable' sets of worlds. $\langle W, P\rangle$ may look like a general frame in modal logic (see Hughes and Cresswell 1996, p. I67), but note that $P$ is not required to satisfy the closure conditions which constrain such general frames. Further the closure conditions apply to indices not to truth sets. The philosophical importance of this is that a restriction on indices is a restriction on how truth is presented, and in a sense is non-linguistic. Restricting truth sets on the other hand may be thought of as a restriction on language. $\langle\mathrm{W}, \mathrm{P}, \mathrm{V}\rangle$ is a model based on $\langle\mathrm{W}, \mathrm{P}\rangle$ iff $\mathrm{V}(\mathrm{p}) \subseteq \mathrm{W}$ for every variable $p$. [p], [^], [ ] and $[\supset]$ are as before except that the indices, $a, b$ etc., are restricted to members of $\mathrm{P}-\mathrm{i}$. e. to the allowable sets of worlds. For $[\mathrm{V} \cup$ all of $\mathrm{a}, \mathrm{b}, \mathrm{c}$ and $\mathrm{b} \cup \mathrm{c}$ must be in $P$, so that $\left[\mathrm{V}^{\cup}\right]$ reads

$\left[V^{\cup}\right] \vDash_{a} \alpha \vee \beta$ iff there are some $b$ and $c$ such that $a, b, c$ and $b \cup c \in P$, and $\mathrm{a} \subseteq \mathrm{b} \cup \mathrm{c}$ and $\vDash_{\mathrm{b}} \alpha$ or $\vDash_{\mathrm{b}} \beta$, and $\vDash_{\mathrm{c}} \alpha$ or $\vDash_{\mathrm{c}} \beta$.

Where $P=\wp W-\emptyset,\langle W, P\rangle$ is called a full tractarian frame, and models based on full frames are equivalent to tractarian models as earlier defined. Full tractarian frames with $\left[\mathrm{V}^{\cup}\right]$ give you classical logic. The logic of general tractarian frames contains intuitionistic logic if $[\mathrm{V}]$ is used, since $\subseteq$ is reflexive and transitive, and the evaluation rules are exact analogues of those used in the Kripke semantics. However, full frames with $[\mathrm{V}]$ are not respectable. If $\left[\mathrm{V}^{\cup}\right]$ is used, the following model will falsify one of the theorems of intuitionistic logic $\sqrt{12}$

$$
((p \supset r) \wedge(q \supset r)) \supset((p \vee q) \supset r)
$$

Let $W=\{1,2,3\}, a=\{2,3\}, b=\{1,2\}, c=\{1,3\}, P=\{a, b, c, b \cup c\}, V(p)=b$, $\mathrm{V}(\mathrm{q})=\mathrm{c}, \mathrm{V}(\mathrm{r})=\emptyset$. In this model $\mathrm{a} \subseteq \mathrm{b} \cup \mathrm{c}$, and $\vDash_{\mathrm{b}} \mathrm{p}$ and $\vDash_{\mathrm{c}} \mathrm{q}$. So $\vDash_{\mathrm{a}} \mathrm{p} \vee \mathrm{q}$. But since $\exists_{a} r$, then $\exists_{a}(p \vee q) \supset r$. Now if $d \subseteq a$ and $d \in P$, then $d=a$, and

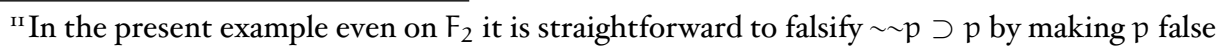
at $i$ and true at $j$ and $k$. In a tractarian frame this would have to be because there are yet more disallowed endpoints, at which $p$ is false.

${ }^{12}$ The following set of axioms for intuitionistic logic comes from Heyting I930:

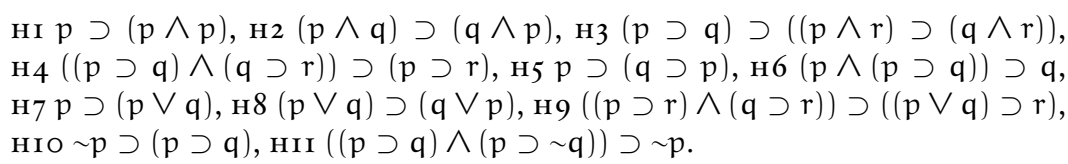

(As I mentioned before I use the 'classical' symbols $\sim$ and $\supset$ in place of $\neg$ and $\rightarrow$.) In the general tractarian semantics using $\left[\mathrm{V}^{\cup}\right]$ all the axioms except $\mathrm{H}_{9}$ will still be valid even if $\left[\mathrm{V}^{\cup}\right]$ is used. All but $\mathrm{H}_{7}, \mathrm{H} 8$ and $\mathrm{H}_{9}$ lack $\mathrm{V}$. $\mathrm{H}_{7}$ only requires the trivial consequence of $\left[\mathrm{V}^{\cup}\right]$ that if $\vDash_{\mathrm{a}} \alpha$ or $\vDash_{\mathrm{a}} \beta$ then $\vDash_{\mathrm{a}} \alpha \vee \beta$, and $\mathrm{H} 8$ simply relies on the fact that $\left[\mathrm{V}^{\cup}\right]$ makes $\vee$ commutative.
} 
since $\exists_{a} p$ and $\exists_{a} q$ then

$$
\exists_{a}((p \supset r) \wedge(q \supset r)) \supset((p \vee q) \supset r) .
$$

To get the class of wff valid in intuitionistic logic $P$ must be restricted in certain ways. Say that a general tractarian frame $\langle\mathrm{W}, \mathrm{P}\rangle$ is $\bigcup$-closed iff for every $\mathrm{C} \subseteq \mathrm{P}$

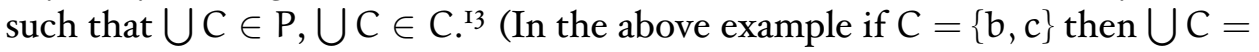
$\mathrm{b} \cup \mathrm{c} \in \mathrm{P}$, but $\mathrm{b} \cup \mathrm{c} \notin \mathrm{C}$.) $\bigcup$-closure may seem philosophically unmotivated, but one can, I think, make at least some progress by considering some comments made on p. 58 of Restall 1999 on the interpretation of disjunction. ${ }^{14}$ Restall notes that the incompleteness of semantic indices causes a problem for the 'classical' rule for disjunction-i.e [V]. For if $a$ is incomplete there seems no reason why it should not provide the information that $\alpha \vee \beta$ is true without either providing the information that $\alpha$ is true, or the information that $\beta$ is true. Restall's answer is that indices are 'maximally specific about their subject matter'. So it might help to look at a simple illustration which I have used in a number of places. ${ }^{15}$ The idea is that 'information' is given by a set B of 'basic particular situations', as it might be by which points of spacetime are occupied and which are not. 'Spacetime' here may be thought of as some kind of 'logical space' or a physical space, though perhaps it is better to think of it as a simplification of a reality which is much more complex. The set $\mathrm{B}$ of all such situations is the set of all pairs of the form $\langle\pi, 1\rangle$ or $\langle\pi, 0\rangle$ where $\pi$ is a spacetime point. $\langle\pi, 1\rangle$ would represent that $\pi$ is occupied, and $\langle\pi, 0\rangle$ that it is not. More generally, where $\Pi$ is any set, the situations structure $B$, strictly $B_{\Pi}$, based on $\Pi$, will be the set of all pairs of the form $\langle\pi, 1\rangle$ or $\langle\pi, 0\rangle$ for $\pi \in \Pi$.

\footnotetext{
${ }^{13} A$ weaker condition will suffice for intuitionistic propositional logic. Say that $\langle W, P\rangle$ is $\bigcup^{*}$ closed iff where $\mathrm{b}, \mathrm{c}$ and $\mathrm{b} \cup \mathrm{c}$ are all in $\mathrm{P}$ then either $\mathrm{b} \subseteq \mathrm{c}$ or $\mathrm{c} \subseteq \mathrm{b}$. $\bigcup^{*}$-closure corresponds precisely with the equivalence of $[\mathrm{V}]$ and $\left[\mathrm{V}^{\cup}\right]$, since if $[\mathrm{V}]$ and $\left[\mathrm{V}^{\cup}\right]$ are equivalent then $\langle\mathrm{W}, \mathrm{P}\rangle$ is $\bigcup^{*}$-closed. For suppose $b, c$ and $b \cup c$ are all allowable but neither $b \subseteq c$ nor $c \subseteq b$. Let $V(p)=b$ and $V(q)=c$. Then $f_{b \cup c} p$ and $\exists_{b \cup c} q$. So if $[V]$ is used then $f_{b \cup c} p \vee q$. But $\mathrm{b} \cup \mathrm{c} \subseteq \mathrm{b} \cup \mathrm{c}$ and $\vDash_{\mathrm{b}} p$ and $\vDash_{\mathrm{c}} \mathrm{q}$, and so if $\left[\mathrm{V}^{\cup}\right]$ is used $\vDash_{\mathrm{b} \cup \mathrm{c}} \mathrm{p} \vee \mathrm{q}$. $\bigcup$-closure implies $\bigcup^{*}$ closure. I. e., if $\langle W, P\rangle$ is $\bigcup$-closed and $a, b$ and $a \cup b \in P$ then either $a \subseteq b$ or $b \subseteq a$, since, given that $\langle W, P\rangle$ is $\bigcup$-closed let $C=\{a, b\}$. Now $a \cup b \in P$ and so $a \cup b \in C$. So $a \cup b=a$ or $a \cup b=b$. So $a \subseteq b$ or $b \subseteq a$. But $\bigcup$-closure is stronger than $\bigcup^{*}$-closure in the sense that there is a tractarian frame $\langle W, P\rangle$ which is $\bigcup^{*}$-closed but not $\bigcup$-closed. For let $W=\{1,2,3\}$ and let $P=\{\{1\},\{2\},\{3\}, W\}$. Then, where $C=\{\{1\},\{2\},\{3\}\}, \cup C \in P$ but $\cup C \notin C$. So $\langle W, P\rangle$ is not $\bigcup$-closed. But if $a \cup b \in P$ then $a=b$ or $a=W$ or $b=W$. Either way $a \subseteq b$ or $b \subseteq a$. (Note that it is crucial that none of $\{1,2\},\{1,3\}$ or $\{2,3\}$ is in $P$.) So $\langle W, P\rangle$ is $\bigcup^{*}$-closed but not U-closed.

${ }^{14}$ Restall is also concerned to allow inconsistent indices, where both $\alpha$ and $\sim \alpha$ might be true, and thus provide a motivation for paraconsistent and relevance logics. A criticism of the interpretation of $\vee$ in relevance logic occurs as early as Copeland 1979, pp. 404-406. Devlin I990 justifies the classical rule for disjunction on p. 90, by saying disjunctive information 'is already one step removed from the way the world is'. On p. 86 he says that while 'the information content of the single infon $\langle\langle$ parent-of, $x, y\rangle\rangle$ is the same as that of the disjunction $\langle\langle$ father-of, $x, y\rangle\rangle \vee\langle\langle$ mother-of, $x, y\rangle\rangle$, 'only the former has infon status'.

${ }^{15}$ The idea is found in Wittgenstein I92I. I first explored a version of it in Cresswell 1972, and followed it up in various ways in Cresswell I973, I990 and 1994.
} 
A situation $\sqrt{16} \mathrm{~s}$ is a subset of $\mathrm{B}$ and will be called consistent if at most one of $\langle\pi, 1\rangle$ and $\langle\pi, 0\rangle$ is in s. Let Con be the set of all consistent situations. Say that any set $a$ of situations is allowable iff there is some $s \in$ Con, call it $s_{a}$, such that $a=\left\{s \in\right.$ Con $\left.: s_{a} \subseteq s\right\}$, and let $\left\langle\right.$ Con, $\left.P_{B}\right\rangle$ be the general tractarian frame in which $P_{B}$ is the set of all allowable $e_{s}$ sets of situations. Intuitively the allowable sets of situations are those which convey precise and full information about a part of the world, that is, those that describe precisely the occupation pattern of some region of space. Such sets are partial since they need speak of only a part of space, but about that region of space they give complete information.

Theorem $3\left\langle\right.$ Con, $\left.\mathrm{P}_{\mathrm{B}}\right\rangle$ is $\bigcup$-closed.

Proof (i) $(\forall s \in C o n)(s \in \bigcup C \equiv(\exists a \in C) s \in a)$.

Suppose $C \subseteq \mathrm{P}_{\mathrm{B}}$ and $\cup \mathrm{C} \in \mathrm{P}_{\mathrm{B}}$. Since $\cup \mathrm{C} \in \mathrm{P}_{\mathrm{B}}$ there is some $\mathrm{s}^{*} \in$ Con such that $\bigcup C=\left\{s \in\right.$ Con $\left.: s^{*} \subseteq s\right\}$, and since $C \subseteq P_{B}$, if $a \in C, a=\{s \in$ Con : $\left.s_{\mathrm{a}} \subseteq s\right\}$. So from (i)

(ii) $(\forall s \in \operatorname{Con})\left(s^{*} \subseteq s \equiv(\exists a \in C) s_{a} \subseteq s\right)$.

and so

$$
\mathrm{s}^{*} \subseteq \mathrm{s}^{*} \equiv(\exists \mathrm{a} \in \mathrm{C}) \mathrm{s}_{\mathrm{a}} \subseteq \mathrm{s}^{*}
$$

So for some $a \in C$,

(iii) $\mathrm{s}_{\mathrm{a}} \subseteq \mathrm{s}^{*}$.

But also from (ii), $(\forall s \in$ Con $)\left((\exists a \in C) s_{a} \subseteq s \supset s^{*} \subseteq s\right)$. So $(\forall s \in$ Con $)(\forall a \in$ C) $\left(s_{a} \subseteq s \supset s^{*} \subseteq s\right)$, and so

$$
(\forall \mathrm{a} \in \mathrm{C})\left(\mathrm{s}_{\mathrm{a}} \subseteq \mathrm{s}_{\mathrm{a}} \supset \mathrm{s}^{*} \subseteq \mathrm{s}_{\mathrm{a}}\right) .
$$

So $s^{*} \subseteq s_{a}$ for every $a \in C$, and so, from (iii) $s^{*}=s_{a}$ for some $a \in C$, and so $a=\bigcup C$ for some $a \in C$, and so $\bigcup C \in C$.

\footnotetext{
${ }^{16}$ The use of 'situations' has been extensively developed in the 'situation semantics' deriving from the work of Jon Barwise and John Perry, and may well have some links with the ideas of the present paper. The principal source for situation semantics is Barwise and Perry 1983. On p. 54, they impose a number of conditions on situations to make them what they call coherent. The conditions include consistency in the sense of the present paper. A more recent survey of work in this tradition is given in Seligman and Moss, 1997. Barwise and Etchemendy, 1990, note that 'infon algebras' are Heyting algebras, and make brief mention of disjunction on p. 50 . The connection between partiality and intuitionistic logic is discussed on p. $44 \mathrm{f}$ of van Benthem 1985. On p. 45 van Benthem notes that the interpretation of $\vee$ in intuitionistic logic may be troublesome in a partial setting. (See also van Benthem I986, pp. 236-239.) My interpretation of indices as sets of worlds betrays a classical bias, and would almost certainly be rejected by most situation theorists.
} 
Theorem 3 might be thought to suggest that the intuitions behind situation semantics support $\bigcup$-closure, and therefore justify intuitionistic logic as its correct logic. However things are not so simple. The original motivation for this semantics was requirement $[\mathrm{A}]$ that a wff $\alpha$ is true at an index $a$ iff $\alpha$ is true at every world in a. But not all situations are worlds, and not all sets of situations are sets of worlds. A world gives complete information about the whole of $\Pi$. A situation $s \in$ Con is a world iff exactly one of $\langle\pi, 1\rangle$ and $\langle\pi, 0\rangle$ is in $s$ for every $\pi \in \Pi$. Let $W_{B}$ be the set of all worlds based on $B$. That is, $w \in W_{B}$ iff $w$ is a world and $w \subseteq B_{\Pi}$. Where $s \in$ Con let $a_{s}=\left\{w \in W_{B}: s \subseteq w\right\}$. That is, $a_{s}$ is the proposition that (set of worlds in which) $s$ is true. Say that any set $a$ of worlds is allowable $w_{w}$ iff $a$ is $a_{s}$ for some $s \in$ Con, and let $\left\langle W_{B}, P_{B}\right\rangle$ be the general tractarian frame in which $W_{B}$ is the set of worlds based on $B_{\Pi}$ and $\mathrm{P}_{\mathrm{B}}$ is the set of all allowable ${ }_{w}$ sets of worlds. It turns out that Theorem 3 no longer holds. Consider the case where $\Pi$ contains just two members $\pi_{1}$ and $\pi_{2}$, and consider the following situations:

$$
\begin{aligned}
& s_{1}=\left\{\left\langle\pi_{1}, 1\right\rangle,\left\langle\pi_{2}, 1\right\rangle\right\}, \\
& s_{2}=\left\{\left\langle\pi_{1}, 1\right\rangle,\left\langle\pi_{2}, 0\right\rangle\right\}, \\
& s_{3}=\left\{\left\langle\pi_{1}, 1\right\rangle\right\} .
\end{aligned}
$$

In this set of situations the worlds are $s_{1}$ and $s_{2}$, and $P_{B}$ contains $\left\{s_{1}\right\},\left\{s_{2}\right\}$ and $\left\{s_{1}, s_{2}\right\}$. But where $C=\left\{\left\{s_{1}\right\},\left\{s_{2}\right\}\right\}$ then $C \subseteq P_{B}$ and $\bigcup C\left(=\left\{s_{1}, s_{2}\right\}\right) \in P_{B}$ but $\cup C \notin C$. This example does not contradict Theorem 3 since, although $\left\{s_{1}, s_{2}\right\}$

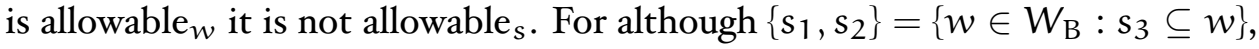
$\left\{s_{1}, s_{2}\right\} \neq\left\{s \in\right.$ Con : $\left.s_{3} \subseteq s\right\}$, since $s_{3} \notin\left\{s_{1}, s_{2}\right\}$. $\left\{s_{1}, s_{2}, s_{3}\right\}$ is allowable $_{s}$ but that is not $\left\{s_{1}\right\} \cup\left\{s_{2}\right\}$.

What this means is that while allowable sets of situations are $\bigcup$-closed, allowable sets of worlds need not be; so that even if we agree that an index gives all and only information about a restricted part of $\Pi$, that still does not decide the question of whether $\bigcup$-closure should be a constraint on the logic of partial information. To take up this question however would require an examination of situation semantics which is beyond the scope of this paper. Hopefully though, the paper presents a slightly different account of how restricting indices to certain sets of worlds provides a semantics which leads to intuitionistic logic in a way which is congenial to those who think that situation theory as described above characterises the form in which information is presented to us, and that we can only make sense of truth and falsity when it is presented in this way.

The next theorem establishes that $[\mathrm{V}]$ and $\left[\mathrm{V}^{\cup}\right]$ give the same results if $\langle W, P\rangle$ is a $U$-closed frame.

Theorem 4 If $\langle W, P\rangle$ is $\bigcup$-closed and $\vee$ is governed by $\left[V^{\cup}\right]$ then $\vDash_{a} \alpha \vee \beta$ iff $\vDash_{a} \alpha$ or $\vDash_{a} \beta$.

Proof Suppose $\vDash_{a} \alpha \vee \beta$. Then there are some $b$ and $c$ such that $b, c$ and $\mathrm{b} \cup \mathrm{c} \in \mathrm{P}, \mathrm{a} \subseteq \mathrm{b} \cup \mathrm{c}, \vDash_{\mathrm{b}} \alpha$ or $\vDash_{\mathrm{b}} \beta$, and $\vDash_{\mathrm{c}} \alpha$ or $\vDash_{\mathrm{c}} \beta$. Given that $\langle\mathrm{W}, \mathrm{P}\rangle$ is 
$\bigcup$-closed let $C=\{b, c\}$. Now $b \cup c \in P$ and so $b \cup c \in C$. So $b \cup c=b$ or $\mathrm{b} \cup \mathrm{c}=\mathrm{c}$. So $\mathrm{a} \subseteq \mathrm{b}$ or $\mathrm{a} \subseteq \mathrm{c}$. If $\mathrm{a} \subseteq \mathrm{b}$ then, by Theorem 2 , since $\vDash_{\mathrm{b}} \alpha$ or $\vDash_{\mathrm{b}} \beta$, $\vDash_{a} \alpha$ or $\vDash_{a} \beta$, and if $a \subseteq c$, since $\vDash_{c} \alpha$ or $\vDash_{c} \beta, \vDash_{a} \alpha$ or $\vDash_{a} \beta$. So in either case $\vDash_{a} \alpha$ or $\vDash_{a} \beta$. Now suppose $\vDash_{a} \alpha$ or $\vDash_{a} \beta$. Since $a \in P$ then $a \cup a \in P$ and $a \subseteq a \cup a$, and so by $\left[V^{\cup}\right], \vDash_{a} \alpha \vee \beta$.

A consequence of Theorem 4 is that although a tractarian frame will satisfy [A] in respect of atomic wff, models based on $\bigcup$-closed frames need not satisfy [A] in respect of complex wff. An approach which is similar to that of the present paper is the Beth-Kripke-Joyal semantics mentioned in footnote I. The similarity arises because, as in the present approach, the indices in this semantics appear construable as something like sets of worlds. But there are important differences. As far as I can tell, when translated into the terminology used in this paper, the $\mathrm{BKJ}$ rule for $\vee$ is

$\left[\vee^{\mathrm{BKJ}}\right] \vDash_{\mathrm{a}} \alpha \vee \beta$ iff there are $\mathrm{b}$ and $\mathrm{c}$ with $\mathrm{a}=\mathrm{b} \cup \mathrm{c}$ and $\vDash_{\mathrm{b}} \alpha$ and $\vDash_{\mathrm{c}} \beta$.

(For a condition similar to $\left[\mathrm{V}^{\mathrm{BKJ}}\right]$ see $\left(^{*}\right)$ on p. 65 of Humberstone 1988.) The principal difference between $\left[\mathrm{V}^{\mathrm{BKJ}}\right]$ and $\left[\mathrm{V}^{\cup}\right]$ is that the condition in the former is conjunctive; it is required that $\vDash_{b} \alpha$ and $\vDash_{c} \beta$. A less essential difference is that the indices in the present paper are non-empty sets of worlds, and there is no reason to suppose that $b o t h \alpha$ and $\beta$ are true anywhere at all. Assuming that the other operators behave in $\mathrm{BKJ}$ just as they do in the present paper then if all sets of worlds, including the empty set, are allowed both $\left[\mathrm{V}^{\mathrm{BKJ}}\right]$ and $\left[\mathrm{V}^{\cup}\right]$ give you classical logic. If no closure conditions at all are added to $\left[\mathrm{V}^{\mathrm{BKJ}}\right]$ then we can falsify $(\sim p \vee \sim p) \supset \sim p$. (Take $W=\{1,2,3\}, a=W, b=\{1,2\}, c=\{1,3\}$ and $d=\{2,3\}$, with $P=\{a, b, c, d\}$. Note that for any $e \in P$, if $e \subseteq b$ then $e=\mathrm{b}$ and if $e \subseteq \mathrm{c}$ then $e=\mathrm{c}$. With $\mathrm{V}(\mathrm{p})=\mathrm{d}$ we have $\vDash_{\mathrm{b}} \sim p$ and $\vDash_{\mathrm{c}} \sim p$, and so by $\left[\vee^{\mathrm{BKJ}}\right], \vDash_{\mathrm{a}} \neg p \vee \neg p$. But $\exists_{\mathrm{a}} \neg p$ since $\mathrm{d} \subseteq \mathrm{a}$ and $\vDash_{\mathrm{d}} p$.) Adding the closure condition of the present paper (in the form described in the note 13) would not help, since it would validate $\neg(p \wedge q) \supset \neg(p \vee q)$. And adding any closure condition to $\left[\mathrm{V}^{\mathrm{BKJ}}\right]$ which yields $[\mathrm{V}]$ will give the closure condition of the present paper. For suppose $a=b \cup c$ and that $a, b$ and $c$ are all allowable. Put $V(p)=b$, and $V(q)=c$. Then $\vDash_{a} p \vee q$. By $[V]$ either $\vDash_{a} p$ or $\vDash_{a} q$. If $\vDash_{a} p$ then $b \cup c \subseteq b$, and if $\vDash_{a}$ q then $b \cup c \subseteq c$; so either $b \subseteq c$ or $c \subseteq$ b. If I understand the claims of the theorems of the Beth-Kripke-Joyal semantics correctly the point appears to be that if you regard principle [A] of the present paper not in terms of classical set theory, but in terms of a local set theory' based on intuitionistic logic, and if you define truth at a as truth at every $w \in$ a then you can prove that $\vee$ satisfies $\left[\mathrm{V}^{\mathrm{BKJ}}\right]$. Notice that it does not follow from [A] that $\vDash_{a} \alpha \vee \beta$ gives either $\vDash_{a} \alpha$ or $\vDash_{a} \beta$. This means that you cannot get $[\mathrm{V}]$ by imposing closure conditions on allowable indices, which is the way intuitionistic logic is obtained in the present paper. You may be able to obtain intuitionistic logic by imposing conditions on truth sets, such as the condition $[\mathrm{EP}]$ described in the text, but as I mentioned in the text, the 
closure conditions of the present paper apply to indices not truth sets. This is in line with my claim to present intuitionistic logic as a matter of how the world presents itself rather than how our language characterises it.

An extended tractarian (general) frame for some fixed language of first-order predicate logic is a quadruple $\langle\mathrm{W}, \mathrm{P}, \mathrm{D}, \mathrm{Q}\rangle$ in which $\mathrm{W}$ is a set of 'worlds', P a non-empty subset of $\wp W-\emptyset$ and $D$ a set of 'individuals'. For every $w \in W$, $\mathrm{Q}(w) \subseteq \mathrm{D}$ is the set of things which 'exist' in $w$. It is required that $\bigcap\{\mathrm{Q}(w)$ : $w \in W\} \neq \emptyset$. If we define $D_{a}$ for $a \in P$ as $\bigcap\{Q(w): w \in a\}$ then since $\bigcap\{\mathrm{Q}(w): w \in W\} \neq \emptyset, \mathrm{D}_{\mathrm{a}}$ will be non-empty, and if $\mathrm{a} \subseteq \mathrm{b}$ then $\mathrm{D}_{\mathrm{b}} \subseteq$ $\mathrm{D}_{\mathrm{a}}$. For a world $w \in W, \mathrm{D}_{w}=\mathrm{Q}(w)$. A model, $\langle\mathrm{W}, \mathrm{P}, \mathrm{D}, \mathrm{Q}, \mathrm{V}\rangle$ based on $\langle\mathrm{W}, \mathrm{P}, \mathrm{D}, \mathrm{Q}\rangle$ adds a function $\mathrm{V}$, where $\mathrm{V}(\varphi)$, for $n$-place $\varphi$, is a set of $\mathrm{n}+1$ tuples of the form $\left\langle u_{1}, \ldots, u_{n}, w\right\rangle$ for $u_{1}, \ldots, u_{n} \in D$ and $w \in W$. In such a model an assignment $\mu$ to the variables is a function such that, for each variable $x, \mu(x) \in D$. Every wff can be given a truth value at an index with respect to an assignment $\mu$. Where $\mu$ and $\rho$ both assign members of the domain $D$ of individuals to the variables I call them $x$-alternatives iff they agree on all variables except (possibly) $x$. Where $\mu$ is any assignment of members of $D$ to the individual variables then

$$
[\varphi] \vDash_{\mathrm{a} \mu} \varphi x_{1} \cdots x_{n} \text { iff }\left\langle\mu\left(x_{1}\right), \ldots, \mu\left(x_{n}\right), w\right\rangle \in \mathrm{V}(\varphi) \text { for every } w \in a .
$$

The rules for $\sim, \wedge, \supset$ and $\vee$ are $[\neg],[\wedge],[\supset]$ and $\left[\mathrm{V}^{\cup}\right]$ (for LPC wff) relativised to an assignment $\mu$. If the indices were worlds the semantic rule for $\forall$ would be

$\left[\forall^{w}\right] \vDash_{w \mu} \forall x \alpha$ iff for every $x$-alternative $\rho$ of $\mu$ such that $\rho(x) \in \mathrm{Q}(\mathcal{w}), \vDash_{w \rho} \alpha$.

For a propositional index $a, \forall$ must be understood to range over the entities in any world in $a$. This dictates the following, for $a, b \in P$ :

$[\forall] \vDash_{a \mu} \forall x \alpha$ iff for every $b \subseteq a$, and every $x$-alternative $\rho$ of $\mu$ such that $\rho(x) \in D_{b}, \vDash_{b \rho} \alpha$.

Theorem 5 If $\langle W, P\rangle$ is full then $\forall$ is classically respectable.

Proof Suppose that $\vDash_{a \mu} \forall x \alpha$ and $w \in a$. Then $\{w\} \subseteq a$, and so, by $[\forall]$, for every $x$-alternative $\rho$ of $\mu$ such that $\rho(x) \in D_{w}, \vDash_{\{w\} \rho} \alpha$. So, by $[\forall], \vDash_{\{w\} \mu} \forall x \alpha$. Suppose $A_{a \mu} \forall x \alpha$. Then for some $b \subseteq a$ there is some $x$-alternative $\rho$ of $\mu$ such that $\rho(x) \in D_{b}$ and $\exists_{b \rho} \alpha$. So by $[A]$ there is some $w \in b$ such that $\exists_{\{w\} \rho} \alpha$. But $\mathrm{D}_{\mathrm{b}} \subseteq \mathrm{D}_{w}$ and so $\rho(x) \in \mathrm{D}_{w}$, and so $\exists_{\{w\} \mu} \forall x \alpha$.

The problem case is, predictably, the existential quantifier. If we take the 'classical' rule we have:

$[\exists] \vDash_{\mathrm{a} \mu} \exists x \alpha$ iff there is some $x$-alternative $\rho$ of $\mu$ such that $\rho(x) \in D_{a}$ and $\vDash_{\mathrm{a} \rho} \alpha$. 
But an operator defined by $[\exists]$ is not respectable. For let $\langle\mathrm{W}, \mathrm{P}\rangle$ be the full frame in which $W=\left\{w_{1}, w_{2}\right\}$, and let $\langle W, P, D, Q, V\rangle$ be the model based on $\langle\mathrm{W}, \mathrm{P}\rangle$ in which $\mathrm{D}=\left\{\mathrm{u}_{1}, \mathrm{u}_{2}\right\}, \mathrm{Q}(w)=\mathrm{D}$ for all $w \in W$ and $\mathrm{V}(\varphi)=$ $\left\{\left\langle u_{1}, w_{1}\right\rangle,\left\langle u_{2}, w_{2}\right\rangle\right\}$. Let $a=W$. Then $\vDash_{\left\{w_{1}\right\} \mu} \exists x \varphi x$ and $\vDash_{\left\{w_{2}\right\} \mu} \exists x \varphi x$. But where $\mu(x)=u_{1}$ and $\rho(x)=u_{2}$ these are the only two assignments for $x$; and $\exists_{\mathrm{a} \mu} \varphi x$ since $\left\langle u_{1}, w_{2}\right\rangle \notin \mathrm{V}(\varphi)$ and $\exists_{\mathrm{a} \rho} \varphi x$ since $\left\langle u_{2}, w_{1}\right\rangle \notin \mathrm{V}(\varphi)$, and so by [ $\exists], \exists_{\mathrm{a} \mu} \exists x \varphi x$ even though $\vDash_{\{w\} \mu} \exists x \varphi x$ for every $w \in a$. The rule for $\exists$ which corresponds to $\left[\mathrm{V}^{\cup}\right]$ is:

$\left[\exists^{*}\right] \vDash_{\mathrm{a} \mu} \exists x \alpha$ iff there is a family $\mathrm{C} \subseteq \mathrm{P}$ such that $\mathrm{a} \subseteq \bigcup \mathrm{C} \in \mathrm{P}$ and, for every $\mathrm{b} \in \mathrm{C}$ there is some $x$-alternative $\rho$ of $\mu$ such that $\rho(x) \in \mathrm{D}_{\mathrm{b}}$ and $\vDash_{\mathrm{b} \rho} \alpha$.

Note that Theorem 2 still holds for all of $[\forall],[\exists]$ and $\left[\exists^{*}\right]$.

Theorem 6 If $\langle\mathrm{W}, \mathrm{P}\rangle$ is full and $\left[\exists^{*}\right]$ is used then $\exists$ is respectable.

Proof Suppose that $\vDash_{\mathrm{a} \mu} \exists x \alpha$. Then there is a family $C \subseteq P$ such that $a \subseteq U C \in P$ and, for every $b \in C$ there is some $x$-alternative $\rho$ of $\mu$ such that $\rho(x) \in D_{b}$ and $\vDash_{b \rho} \alpha$. Suppose $w \in a$. Then $w \in \bigcup C$ and so, for some $\mathrm{b} \in \mathrm{C}, w \in \mathrm{b}$. So, by $[\mathrm{A}], \vDash_{\{w\} \rho} \alpha$. Now $\mathrm{D}_{\mathrm{b}} \subseteq \mathrm{D}_{w}$ and so $\rho(\mathrm{x}) \in \mathrm{D}_{w}$ and so $\vDash_{\{w\} \mu} \exists x \alpha$. Suppose $\vDash_{\{w\} \mu} \exists x \alpha$ for every $w \in$ a. Then $\vDash_{\{w\} \rho} \alpha$ for some $x-$ alternative $\rho$ of $\mu$ such that $\rho(x) \in D_{w}$. Let $C=\{\{w\}: w \in a\}$. Then $a \subseteq \cup C$ and for every $b \in C$ there is some $x$-alternative $\rho$ of $\mu$ such that $\rho(x) \in D_{b}$ and $\vDash_{\mathrm{b} \rho} \alpha$. So, by $\left[\exists^{*}\right], \vDash_{\mathrm{a} \mu} \exists x \alpha$.

A wff $\alpha$ is valid on an extended tractarian frame $\langle W, P, D, Q\rangle$ iff $\alpha$ is valid in every model $\langle W, P, D, Q, V\rangle$ based on $\langle W, P, D, Q\rangle$, i. e, iff $\vDash_{a \mu} \alpha$ for every $a \in P$ and every $\mu$ such that $\mu(x) \in D_{a}$ for every $x$ free in $\alpha$. A frame (model) is said to be a frame for (model for) a set $\mathrm{X}$ of wff iff every $\alpha \in \mathrm{X}$ is valid on that frame (model). Theorems 5 and 6 guarantee the link with classical logic. A (classical) interpretation for LPC is a pair $\langle\mathrm{D}, \mathrm{V}\rangle$ where $\mathrm{D}$ is a non-empty class, and $V$ is a function such that where $\varphi$ is an $n$-place predicate then $V(\varphi)$ is a class of $n$-tuples from $D$. Truth is defined with respect to an assignment $\mu$ to the variables in the following way:

- $V_{\mu}\left(\varphi x_{1} \ldots x_{n}\right)=1$ if $\left\langle\mu\left(x_{1}\right), \ldots, \mu\left(x_{n}\right)\right\rangle \in V(\varphi)$ and 0 otherwise.

- $V_{\mu}(\alpha \wedge \beta)=1$ if $V_{\mu}(\alpha)=1$ and $V_{\mu}(\beta)=1$ and 0 otherwise.

- $V_{\mu}(\sim \alpha)=1$ if $V_{\mu}(\alpha)=0$ and 0 otherwise.

- $V_{\mu}(\alpha \vee \beta)=1$ if $V_{\mu}(\alpha)=1$ or $V_{\mu}(\beta)=1$ and 0 otherwise.

- $V_{\mu}(\alpha \supset \beta)=1$ if either $V_{\mu}(\alpha)=0$ or $V_{\mu}(\beta)=1$ and 0 otherwise.

- $V_{\mu}(\forall x \alpha)=1$ if $V_{\rho}(\alpha)=1$ for every $x$-alternative $\rho$ of $\mu$, and 0 otherwise.

- $V_{\mu}(\exists x \alpha)=1$ if there is an $x$-alternative $\rho$ of $\mu$ such that $V_{\rho}(\alpha)=1$, and 0 otherwise. 
A wff $\alpha$ is valid in a classical interpretation $\langle D, V\rangle$ iff $V_{\mu}(\alpha)=1$ for every assignment $\mu$. A wff $\alpha$ is a classical consequence of a set $X$ of wff iff $\alpha$ is valid in every classical interpretation in which every member of $X$ is valid.

Suppose that $\langle W, P\rangle$ is a full tractarian frame and that $\langle W, P, D, Q, V\rangle$ is any tractarian model based on $\langle W, P\rangle$. For any $w \in W$, let $\left\langle\mathrm{Q}(w), V^{w}\right\rangle$ be the classical interpretation in which $\left\langle u_{1}, \ldots, u_{n}\right\rangle \in V^{w}(\varphi)$ iff $\left\langle u_{1}, \ldots, u_{n}, w\right\rangle \in$ $V(\varphi)$. An obvious induction on the construction of $\alpha$ establishes, for every assignment $\mu$ where $\mu(x) \in Q(w)$ for every $x$ free in $\alpha$,

Theorem $7 \mathrm{~V}_{\mu}^{\mathcal{w}}(\alpha)=1 \mathrm{iff} \vDash_{\{w\} \mu} \alpha$.

Conversely, suppose that $\langle\mathrm{D}, \mathrm{V}\rangle$ is a classical interpretation and let $w$ be some arbitrary object and let $\mathrm{Q}(w)=\mathrm{D}$, and let $\left\langle\mathrm{W}, \mathrm{P}, \mathrm{D}, \mathrm{Q}, \mathrm{V}^{*}\right\rangle$ be the (full) tractarian model in which $W=\{w\}, P=\{\{w\}\}$, and for $u_{1}, \ldots, u_{n} \in D$, we have $\left\langle u_{1}, \ldots, u_{n}, w\right\rangle \in V^{*}(\varphi)$ iff $\left\langle u_{1}, \ldots, u_{n}\right\rangle \in V(\varphi)$. Then, also by induction:

Theorem $8 \quad V_{\mu}(\alpha)=1$ iff $\vDash_{\{w\} \mu} \alpha$.

Where $X$ is any set of wff say that $X \vDash_{T^{+}} \alpha$ iff $\alpha$ is valid in every full tractarian model for $X$.

Theorem $9 X \vDash_{T^{+}} \alpha$ iff $\alpha$ is a classical consequence of $X$.

Proof Suppose that $X f_{T^{+}} \alpha$. Then it follows that there is a full tractarian model $\langle W, P, D, Q, V\rangle$ in which every $\beta \in X$ is valid but $f_{a \mu} \alpha$ for some $a \in P$ and some assignment $\mu$ such that $\mu(x) \in D_{a}$ for every $x$ free in $\alpha$. Since $\langle W, P\rangle$ is full all the operators are respectable, and so, by $[\mathrm{A}]$, for some $w \in \mathrm{a}, \exists_{\{w\} \mu} \alpha$, and since $\mu(x) \in D_{a}$ and $\{w\} \subseteq a$ then $\mu(x) \in Q(w)\left(=D_{\{w\}}\right)$. Since every $\beta \in X$ is valid in $\langle W, P, D, Q, V\rangle$ then $\vDash_{\{w\} \rho} \beta$ for every $\beta \in X$ and every $\rho$ such that $\rho(x) \in Q(w)$ for every $x$ free in $\beta$. So, by Theorem $7, V_{\mu}^{w}(\alpha)=0$ and $V_{\rho}^{w}(\beta)=1$, for every $\beta \in X$ and every assignment $\rho$ such that $\rho(x) \in$ $Q(w)$ for every $x$ free in $\beta$. But then $\alpha$ is not a classical consequence of $X$. Conversely, suppose that $\alpha$ is not a classical consequence of $X$. Then there is a classical interpretation $\langle D, V\rangle$ in which $V_{\mu}(\alpha)=0$ for some assignment $\mu$, and $V_{\rho}(\beta)=1$ for every $\beta \in X$ and every assignment $\rho$. Where $\left\langle W, P, D, Q, V^{*}\right\rangle$ is defined as in Theorem $8, \exists_{\{w\} \mu} \alpha$ where $\mu(x) \in D_{w}$ for every $x$ free in $\alpha$, and $\vDash_{\{w\} \rho} \beta$ for every $\beta \in X$ and every $\rho$ such that $\rho(x) \in D_{w}$ for every $x$ free in $\beta$, and so $\alpha$ fails in a full tractarian model for $X$, and so $X=_{T^{+}} \alpha$.

To obtain intuitionistic logic we require $\bigcup$-closed frames. The next theorem shews that $[\exists]$ and $\left[\exists^{*}\right]$ are equivalent in a $\bigcup$-closed tractarian frame.

Theorem io If $\langle W, P\rangle$ is $\bigcup$-closed and $\left[\exists^{*}\right]$ is used then $\vDash_{a \mu} \exists x \alpha$ iff there is some $x$-alternative $\rho$ of $\mu$ such that $\rho(x) \in D_{a}$ and $\vDash_{a} \rho$.

Proof Suppose $\left[\exists^{*}\right]$ is used and $\vDash_{\mathrm{a} \mu} \exists x \alpha$. Then there is a family $C \subseteq \mathrm{P}$ such that $a \subseteq \cup C \in P$ and, for every $b \in C$ there is some $x$-alternative $\rho$ of 
$\mu$ such that $\rho(x) \in D_{b}$ and $\vDash_{b \rho} \alpha$. Since $\langle W, P\rangle$ is $U$-closed then $\bigcup C \in C$ and so there is some $x$-alternative $\rho$ of $\mu$ such that $\rho(x) \in D_{\cup C}$ and $\vDash_{\cup C} \rho$. But $a \subseteq \cup C$ and so $D_{\cup C} \subseteq D_{a}$. So $\rho(x) \in D_{a}$ and, by Theorem $2, \vDash_{a} \alpha$. If $\vDash_{a \rho} \alpha$ for some $x$-alternative $\rho$ of $\mu$ let $C=\{a\}$. Then $\bigcup C=a$ and so $\mathrm{a} \subseteq \bigcup C$, and for every $\mathrm{b} \in \mathrm{C}, \vDash_{\mathrm{b} \rho} \alpha$. So $\vDash_{\mathrm{a} \mu} \exists x \alpha$.

A Kripke model for intuitionistic predicate logic is a quintuple $\langle I, R, D, Q, V\rangle$ in which $I$ is a set of 'indices', $R$ a reflexive, transitive and antisymmetrical relation on $I, D$ another set, and $Q$ a function such that for $i \in I, Q(i) \subseteq D$ and if $i R j$ then $Q(i) \subseteq Q(j) .{ }^{17} V$ is a function such that, where $\varphi$ is an $n$ place predicate, $V(\varphi)$ is a set of $n+1$-tuples each of the form $\left\langle u_{1}, \ldots, u_{n}, i\right\rangle$ for $u_{1}, \ldots, u_{n} \in D$ and $i \in I$, where, if $\left\langle u_{1}, \ldots, u_{n}, i\right\rangle \in V(\varphi)$ and $i R j$ then $\left\langle u_{1}, \ldots, u_{n}, j\right\rangle \in V(\varphi)$. For atomic wff $\varphi x_{1} \cdots x_{n}$ we have $V_{\mu}\left(\varphi x_{1} \cdots x_{n}, i\right)=$ 1 if $\left\langle\mu\left(x_{1}\right), \ldots, \mu\left(x_{n}\right), i\right\rangle \in V(\varphi)$ and 0 otherwise. The propositional operators work as before, though relativised to an assignment $\mu$. The quantifiers are evaluated as

$\left[\forall^{\mathrm{I}}\right] V_{\mu}(\forall x \alpha, i)=1$ if $V_{\rho}(\alpha, j)=1$ for every $j$ such that $i R j$, and every $x$ alternative $\rho$ of $\mu$ such that $\rho(x) \in Q(j)$ and 0 otherwise ${ }^{18}$

$\left.{ }^{[}{ }^{\mathrm{I}}\right] \mathrm{V}_{\mu}(\exists x \alpha, \mathfrak{i})=1$ if $\mathrm{V}_{\rho}(\alpha, i)=1$ for at least one $x$-alternative $\rho$ of $\mu$ such that $\rho(x) \in Q(i)$ and 0 otherwise.

A wff is valid in a model $\langle I, R, D, Q, V\rangle$ iff for every index $i \in I, V_{\mu}(\alpha, i)=1$ for every assignment $\mu$ such that $\mu(x) \in Q(i)$ for every variable $x$. I shall call $\langle I, R, D, Q\rangle$ an extended Kripke frame; and validity on a frame will mean validity in every model based on that frame.

I now shew that restricting tractarian frames to those which are $U$-closed gets intuitionistic logic. Let $\langle I, R\rangle$ be an intuitionistic frame. For $i \in I$ let $i^{+}=\{j: i R j\}$. Then $i R j$ iff $j^{+} \subseteq i^{+}$. Let $\left\langle I, P^{I}\right\rangle$ be the tractarian frame obtained from $\langle I, R\rangle$ by letting $a \in P^{I}$ iff $a=i^{+}$for some $i \in I$.

Theorem in If $C \subseteq \mathrm{P}^{\mathrm{I}}$ and $\bigcup \mathrm{C} \in \mathrm{P}^{\mathrm{I}}$ then $\bigcup \mathrm{C} \in \mathrm{C}$.

Proof First note that if $C \subseteq \mathrm{P}^{\mathrm{I}}$ and $a \in C$ then $a=i^{+}$for some $i \in I$ (call it $i_{a}$ ); and, in particular, if $\bigcup C \in \mathrm{P}^{\mathrm{I}}$ then $\bigcup C=i_{*}^{+}$for some $i_{*} \in \mathrm{I}$. (I.e.

\footnotetext{
${ }^{17}$ See Kripke 1965. (The semantic rules for the quantifiers are on p. 96.) For the antisymmetry condition see the comment on Theorem 2 above.

${ }^{18}$ It might be instructive to consider what would happen if the following rule were used for $\forall$ :

$\left[\forall^{\prime}\right] \quad V_{\mu}(\forall x \alpha, i)=1$ if $V_{\rho}(\alpha, i)=1$ for every $x$-alternative $\rho$ of $\mu$ such that $\rho(x) \in Q(i)$ and 0 otherwise.

By $\left[\forall^{\prime}\right]$, given Theorem $2, \forall x \alpha$ would be true at $i$ iff at every $j$ such that $i R j, \alpha$ is true of everything which exists in $i$. The difference between $\forall$ according to $\left[\forall^{\mathrm{I}}\right]$ and according to $\left[\forall^{\prime}\right]$ mimics the difference in ordinary modal logic between $\square \forall x \alpha$ and $\forall x \square \alpha$, except that in modal logic the difference can be expressed syntactically. In tractarian models $\left[\forall^{\prime}\right]$ would be what you get if $[\forall]$ were to read:

$\vDash_{\mathrm{a} \mu} \forall x \alpha$ iff for every $x$-alternative $\rho$ of $\mu$ such that $\rho(x) \in D_{a}, \vDash_{a} \rho$.
} 
$i_{*}=i_{\cup C}$.) Now, for any $j \in I, j \in i_{*}^{+}$iff for some $a \in C, j \in a$. So in particular $i_{*} \in i_{*}^{+}$iff for some $a \in C, i_{*} \in$ a. But $i^{*} \in i_{*}^{+}$and so $i^{*} \in$ a; i.e., $i_{a} R i_{*}$. But $i_{a} \in \bigcup C$, i. e $i_{a} \in i_{*}^{+}$, and so $i_{*} R i_{a}$. So, by antisymmetry $i_{*}=i_{a}$ and so $i_{*}^{+}=a$, for some $a \in C$. So $\cup C \in C,[9]$

Let $\langle I, R\rangle$ be an intuitionistic frame generated by an index $i^{*}$, and then let $\langle I, R, D, Q, V\rangle$ be an intuitionistic model based on $\langle I, R\rangle$. Consider the corresponding tractarian model $\left\langle\mathrm{I}, \mathrm{P}^{\mathrm{I}}, \mathrm{D}, \mathrm{Q}, \mathrm{V}\right\rangle$, based on $\left\langle\mathrm{I}, \mathrm{P}^{\mathrm{I}}\right\rangle$. Note that $\mathrm{Q}(\mathrm{i})=$ $D_{i^{+}}$as $D_{i^{+}}$is defined for tractarian models, since $u \in D_{i^{+}}$iff $u \in Q(j)$ for every $j \in \mathfrak{i}^{+}$, i.e. every $j$ such that $i R j$; and given that $Q(i) \subseteq Q(j)$ this will hold iff $u \in Q(i)$. Furthermore, $\bigcap\{\mathrm{Q}(i): i \in I\}=\mathrm{Q}\left(i^{*}\right)$, and it follows that $\bigcap\{\mathrm{Q}(\mathrm{i}): i \in \mathrm{I}\} \neq \emptyset$.

Theorem I $2 \vDash_{i^{+} \mu} \alpha$ iff $V_{\mu}(\alpha, i)=1$.

Proof Suppose $\vDash_{i^{+} \mu} \varphi x_{1} \ldots x_{n}$. Then $\left\langle\mu\left(x_{1}\right), \ldots, \mu\left(x_{n}\right), j\right\rangle \in V(\varphi)$ for all $j \in i^{+}$, i. e., for all $j$ such that $i R j$. In particular, $\left\langle\mu\left(x_{1}\right), \ldots, \mu\left(x_{n}\right), i\right\rangle \in$ $V(\varphi)$, and so $V_{\mu}\left(\varphi x_{1} \ldots x_{n}, i\right)=1$. Suppose $V_{\mu}\left(\varphi x_{1} \ldots x_{n}, i\right)=1$. Then $\left\langle\mu\left(x_{1}\right), \ldots, \mu\left(x_{n}\right), j\right\rangle \in V(\varphi)$ for all $j$ such that $i R j$, i. e., for all $j \in i^{+}$. So $\vDash_{i^{+} \mu}$ $\varphi x_{1} \ldots x_{n}$. The rules for $\sim, \wedge, \supset$ and $\forall$ are exactly parallel for intuitionistic models and tractarian models and so the induction will go through. This can be illustrated in the case of $\neg$ : If $\vDash_{i^{+} \mu} \sim \alpha$ then $\exists_{b \mu} \alpha$ for every $b \in P$ such that $b \subseteq i^{+}$. Since $b \in P, b=j^{+}$for some $j \in I$, and $f_{j^{+} \mu} \alpha$. So, by the induction hypothesis, $V_{\mu}(\alpha, j)=0$. Since $j^{+} \subseteq i^{+}, i R j$; and so $V_{\mu}(\alpha, j)=0$ for every $j$ such that $i R j$, and so $V_{\mu}(\sim \alpha, i)=1$. If $f_{i^{+} \mu} \sim \alpha$ then there is some $j^{+} \subseteq i^{+}$such that $\vDash_{j^{+} \mu} \alpha$; and so $V_{\mu}(\alpha, j)=1$ and $i R j$. So $V_{\mu}(\sim \alpha, i)=0$. The induction for $\vee$ and $\exists$ is exactly parallel if [V] and $[\exists]$ are used in the tractarian model. But by Theorem II, the model is $\bigcup$-closed and so, by Theorems 4 and IO, the result is the same if $\left[\mathrm{V}^{\cup}\right]$ and $\left[\exists^{*}\right]$ are used.

For the other direction, where $\langle W, P, D, Q, V\rangle$ is a tractarian model and $\langle W, P\rangle$ is $\bigcup$-closed, and $R$ is the converse of $\subseteq$, and for $a \in P, Q^{*}(a)=\bigcap\{Q(w)$ : $w \in a\}$, then $\left\langle P, R, D, Q^{*}, V\right\rangle$ is an intuitionistic model for which the following holds for every wff $\alpha$ and every $a \in P$ :

Theorem $\mathrm{I} 3 \vDash_{\mathrm{a} \mu} \alpha$ iff $\mathrm{V}_{\mu}(\alpha, a)=1$.

Proof The proof, given that $\langle W, P\rangle$ is $\bigcup$-closed, is, as for Theorem I2, by induction on the construction of $\alpha$.

\footnotetext{
${ }^{19} \mathrm{P}^{\mathrm{I}}$ sets are $\bigcup$-closed and therefore $\cup^{*}$ closed in the sense of footnote $\mathrm{I} 3$ which speaks of allowable unions of indices. It is also instructive to consider their intersection. Suppose that $i^{+} \cap j^{+}$is non-empty. Then there is some $k$ such that $k \in i^{+} \cap j^{+}$. But then $i R k$ and $j R k$. So if $i^{+} \cap j^{+}$is non-empty there is some $k$ such that $i R k$ and $j R k$. The condition that any two indices can see a common index marks off the extension of intuitionistic logic obtained by adding $\sim p \vee \sim \sim p$. (See Dummett and Lemmon 1959, p. 252.) In a finite frame the condition entails that there is a 'bottom' index $-a k^{*} \in I$ such that $i R k^{*}$ for all $i \in I$. Further $[\sim]$ entails that in any frame with a bottom index $k^{*}$, for any $i \in I$ and any wff $\alpha, V(\sim \alpha, i)=V\left(\sim \alpha, k^{*}\right)$. What this means is that in any finite frame, on the assumption that every pair of allowable propositions has a non-empty intersection, every negated wff behaves completely classically.
} 
It is an immediate consequence of Theorems $\mathrm{I} 2$ and $\mathrm{I} 3$ that a wff fails in an intuitionistic model iff it fails in a $\bigcup$-closed tractarian model. This shews that the class of wff valid in intuitionistic logic is just the class of wff valid on $U$ closed extended tractarian frames. Further, Theorems I2 and I3 guarantee the equivalence of 'tractarian' logic and intuitionistic logic in the following strong sense:

THEOREM I 4 Where $X$ is any set of wff of LPC, and $\alpha$ is any wff and $X \vDash_{T \cup} \alpha$ means that $\alpha$ is valid in every $\bigcup$-closed tractarian model for $X$, and $X \vDash_{I} \alpha$ means that $\alpha$ is valid in every Kripke model for $X$, then $X \vDash_{T} \cup \alpha$ iff $X \vDash_{I} \alpha$

I am not able to claim that this semantics is superior to the many that are already on offer for intuitionistic logic, but insofar as it seems a little different, and has some philosophical motivation, it is perhaps worthy of consideration.

REFERENCES

Barwise J., and J. Etchemendy, 1990, 'Information, infons and inference.' In Cooper, R., K. Mukai and J. Perry (editors), Situation Theory and Applications, Volume I, Stanford, CSLI, pp. 33-78.

Barwise, J., and J. Perry, 1983, Situations and Attitudes, Cambridge Mass, m Iт Press.

Bell, J. L., 1988, Toposes and Local Set Theories: An Introduction, Oxford, Clarendon Press.

Benthem, J. F. A. K. van, 1985, A Manual of Intensional Logic, Stanford, CSLI Publications.

No II 4 , pp. 225-246.

1986, 'Partiality and nonmonotonicity in classical logic.' Logique et Analyse,

Copeland, B. J., 1979, 'On when a semantics is not a semantics: some reasons for disliking the Routley-Meyer semantics for relevance logic.' Fournal of Philosophical Logic, Vol. 8, pp. 399-4I3.

Cresswell, M. J., 1972, 'The world is everything that is the case.' Australasian fournal of Philosophy, Vol 50, pp. I-13.

1973, Logics and Languages, London, Methuen.

1990, Entities and Indices, Dordrecht, Kluwer.

1994, Language in the World, Cambridge, Cambridge University Press.

Dalen, D. van, 1986, 'Intuitionistic logic.' In Gabbay D.M., and F. Guenthner (editors), Handbook of Philosophical Logic, Dordrecht, Reidel, Vol. III, Ch. 4, pp. 225-339

Devlin, K., 1990, 'Infons and types in an information-based logic.' In Cooper, R., K. Mukai and J. Perry, (editors), Situation Theory and Applications, Vol I, Stanford, CsLI Publications 1990, pp. 79-95

Došen, K., I99I, 'Kripke models and nonhereditary Kripke models, for the Heyting Propositional Calculus.' Notre Dame fournal of Formal Logic, Vol 32, pp. 580-597 
Dummett, M. A. E. and E. J. Lemmon, 1959, 'Modal logics between $\mathrm{S}_{4}$ and S5.'

Zeitschrift für mathematische Logik und Grundlagen der Mathematik, Vol 5, pp. $250-264$

Gödel, K., I933, 'Eine Interpretation des intuitionistischen Aussagenkalküls.'

Ergebnisse eines mathematischen Kolloquims Vol 4, pp. 34-40

Heyting, A., I930, 'Die formalen Regeln der intuitionistischen Logik.' Sitzungsberichte der Preussischen Akademie der Wissenschaften, Physikalische-mathematische Klasse, pp. $42-56$

Hughes, G. E., and M. J. Cresswell, 1996, A New Introduction to Modal Logic, London, Routledge

Humberstone, I. L., 198I, 'From worlds to possibilities.' fournal of Philosophical Logic Vol Io, pp. 313-34I.

I988, 'Operational semantics for positive R.' Notre Dame fournal of Formal

Logic, Vol 29, pp. 6I-80

Kripke, S. A., 1965, 'Semantical analysis of intuitionistic logic I.' In Crossley, J. N. and M. A. E. Dummett (editors), Formal Systems and Recursive Functions, Amsterdam,

North Holland Publishing Co., 92-I29

Lambek, J., Scott, P. J., I986, Introduction to Higher Order Categorical Logic, Cambridge, Cambridge University Press.

Restall, G, 1999, 'Negation in relevant logics.' In Gabbay, D. M., and H. Wansing (editors), What is Negation?, Dordrecht, Kluwer Academic Publishers, pp. 53-76

Schulz, S. M., 1993, 'Modal situation theory.' In Aczel, P., D. Israel, Y. Katigiri and S.

Peters (editors), Situation Theory and Applications, Vol 3, Stanford, csli Publications, pp. I63-I88

Seligman, J., and L. S. Moss, I997, 'Situation theory.' In Benthem, van, J. and A. ter Meulen (editors), Handbook of Logic and Language, Amsterdam, Elsevier/mit Press, pp. 239-309

Wittgenstein, L., I921, Tractatus Logico-Philosophicus (translated by D. F. Pears and B. F. McGuinness), second printing 1963. London, Routledge and Kegan Paul. 
The Australasian fournal of Logic (ISSN I448-5052) disseminates articles that significantly advance the study of logic, in its mathematical, philosophical or computational guises. The scope of the journal includes all areas of logic, both pure and applied to topics in philosophy, mathematics, computation, linguistics and the other sciences.

Articles appearing in the journal have been carefully and critically refereed under the responsibility of members of the Editorial Board. Only papers judged to be both significant and excellent are accepted for publication.

The journal is freely available at the journal website at

$$
\text { http://www.philosophy.unimelb.edu.au/ajl/ }
$$

All issues of the journal are archived electronically at the journal website.

SUBSCRIPTIONS Individuals may subscribe to the journal by sending an email, including a full name, and email address and a postal address to the managing editor at ajl-editors@unimelb.edu.au Subscribers will receive email abstracts of accepted papers to an address of their choice. For institutional subscription, please email the managing editor at ajl-editors@unimelb.edu.au.

Complete published papers may be downloaded at the journal's website at http://www.philosophy.unimelb.edu.au/ajl/ The journal currently publishes in pdf format.

Submission The journal accepts submissions of papers electronically. To submit an article for publication, send the ${ }^{\Delta} \mathrm{T}_{\mathrm{E}} \mathrm{X}$ source of a submission to a member of the editorial board. For a current list of the editorial board, consult the website.

The copyright of each article remains with the author or authors of that article. 